\title{
Ordenación y gestión integrada de cuencas hidrográficas con altos índices erosivos en el ámbito árido sudcaliforniano. Caso particular Cuenca de La Paz en Baja California Sur, México
}

\author{
Z. Flores M. ${ }^{* 1}$, Torrent J. ${ }^{1}$, López $\mathrm{L}^{1}$ \\ ${ }^{1}$ Departamento de Ingeniería Hidráulica y Medio Ambiente. Sección interdepartamental Forestal. Universitat \\ Politècnica de València. Camino de Vera s/n 46022 Valencia. \\ *e-mail: maflolo1@posgrado.upv.es
}

\section{Resumen}

La ordenación de cuencas hidrográficas en un contexto actual debe dar respuesta a los diferentes escenarios de actuación desarrollados por la población, de una forma sistémica y sostenible. Es así como el presente estudio trabaja con la cuenca de La Paz en el estado de Baja California Sur (BCS), de la Península de Baja California, México. Esta cuenca es un reflejo típico del entorno árido sudcaliforniano; cuenta con la única presencia de cursos de agua efímeros (arroyos), en un territorio que es proclive a la afección por huracanes, presenta una cubierta vegetal expuesta a un excesivo pastoreo que produce una escorrentía con elevado arrastre de sedimentos, ocasionando la degradación del suelo en la parte alta de la cuenca y un consecuente cambio de curso de los arroyos aguas abajo. Esto plantea un panorama urgente de atender: gran erosión del área de estudio, la vegetación es escasa y difícil de conservar, los frecuentes periodos de sequía no han sido estudiados como para prever contingencias, la demanda hídrica en la Ciudad de La Paz (capital de BCS) es cada vez mayor y existe una clara desigualdad en la distribución y gestión pluviométrica de la zona. Se aplicarán tres metodologías clásicas de ordenación de cuencas hidrográficas en el terreno árido sudcaliforniano con el objetivo de cuantificar los niveles erosivos de la zona, así como proponer posibles escenarios futuros de actuación y cambios de uso de suelo en la región.

Palabras clave: Erosión, metodologías ordenación cuencas, restauración hidrológico forestal, sequía, SIG. 


\section{Introducción}

La ordenación de una cuenca hidrográfica se centra en el estudio del estado físico de la cuenca vertiente y en el análisis de su previsible comportamiento ante los eventos torrenciales, tanto ordinarios como extraordinarios, todo esto con el propósito de conocer cómo es realmente la cuenca y cómo se comporta; así como para prever su evolución y detectar sus carencias, a fin de proponer las medidas pertinentes para tratar de subsanarlas.

Los escenarios derivados del uso inadecuado de los recursos naturales y de actividades no planificadas mayoritariamente provocan la desestabilización de un sistema natural, precisamente por no considerar previamente la conexión existente entre los distintos elementos que interaccionan en una cuenca hidrográfica. Este tipo de escenarios son recurrentes en la zona Noroeste del territorio mexicano, principalmente en la zona de estudio de la presente investigación.

La Península de Baja California está ubicada en la región Noroeste de la República Mexicana. En ella se ubica la cuenca de La Paz, en la parte Suroriental de la Península de Baja California (Fig. 1). Tiene una superficie total de $1221 \mathrm{~km}^{2}$ y presenta una forma cercana a la circular. En la cuenca de La Paz predominan los ecosistemas desértico y árido. La precipitación es poco frecuente y alcanza un promedio de sólo $180 \mathrm{~mm}$ al año, la más baja de entre todos los estados de México y la cual en su mayoría es proveniente de los huracanes (Z. Flores 1998). Dada la enorme importancia que tiene el agua para los ciclos funcionales de animales y plantas en una región tan árida como lo es el estado de Baja California Sur, es conveniente pensar en la lluvia como un agente determinante al momento de plantear posibles modificaciones a los usos del suelo en la zona de estudio.

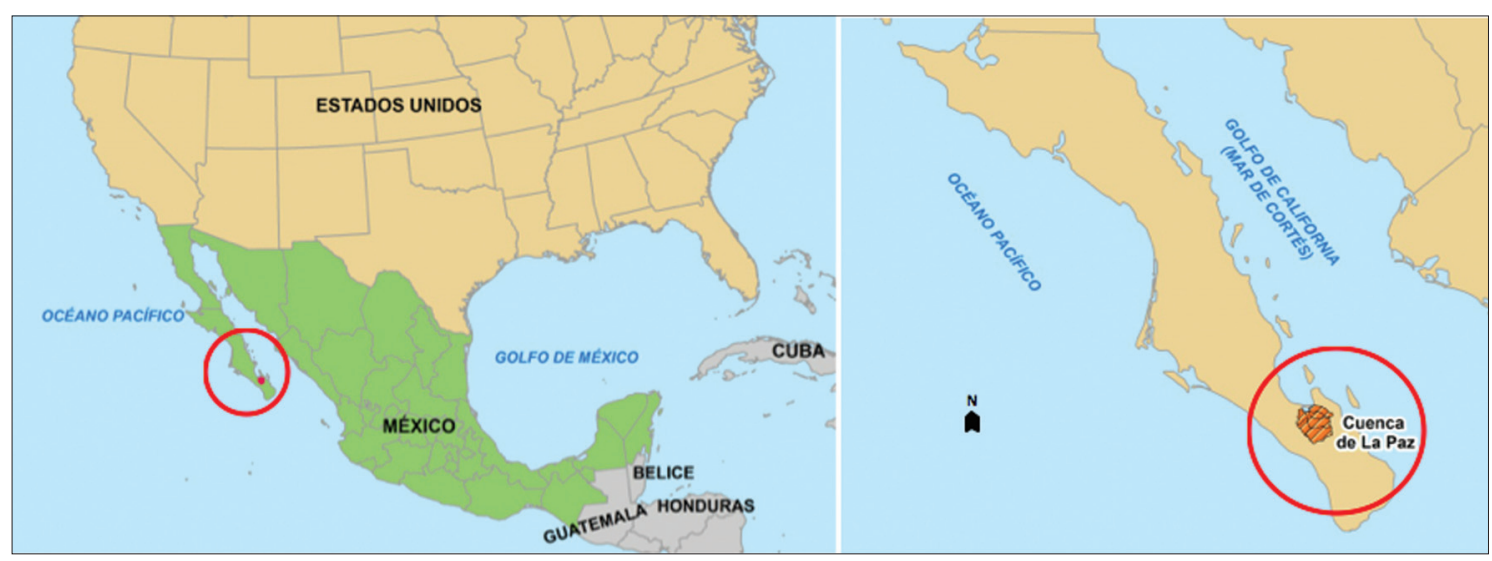

Figura 1. Ubicación de la Cuenca de La Paz en Norteamérica (izquierda) y México (derecha).

En base al origen geológico, la litología, las condiciones climáticas y el relieve presente en la zona de estudio, se han determinado suelos de escaso desarrollo genético y muy susceptibles a la remoción de sus partículas. En la mayor parte de 
los terrenos de la Cuenca de La Paz se desarrollan matorrales xerófilos, como los sarcocaule, sarcocrasicaule, desértico micrófilo, crasicaule y mezquital (Sánchez, 2004)

Las superficies dedicadas a la agricultura ocupan zonas de declive suave. El principal limitante para el desarrollo agropecuario es la escasez de agua. La cuenca de La Paz no tiene ríos o corrientes de aguas superficiales permanentes, situación que se repite a lo largo de todo el estado de Baja California Sur. La única fuente confiable de agua es la subterránea, acotada al acuífero de La Paz, que abastece a ciudades como La Paz (capital de Baja California Sur con 215178 habitantes según el censo poblacional INEGI 2010) y otras (Fig. 2). Lo anterior plantea un horizonte urgente de atender a una correcta ordenación de los recursos de la cuenca y especialmente los del ciclo hidrológico, pues los requerimientos de agua de la Ciudad de La Paz son cada vez mayores y demandan un real interés en el estudio de la zona

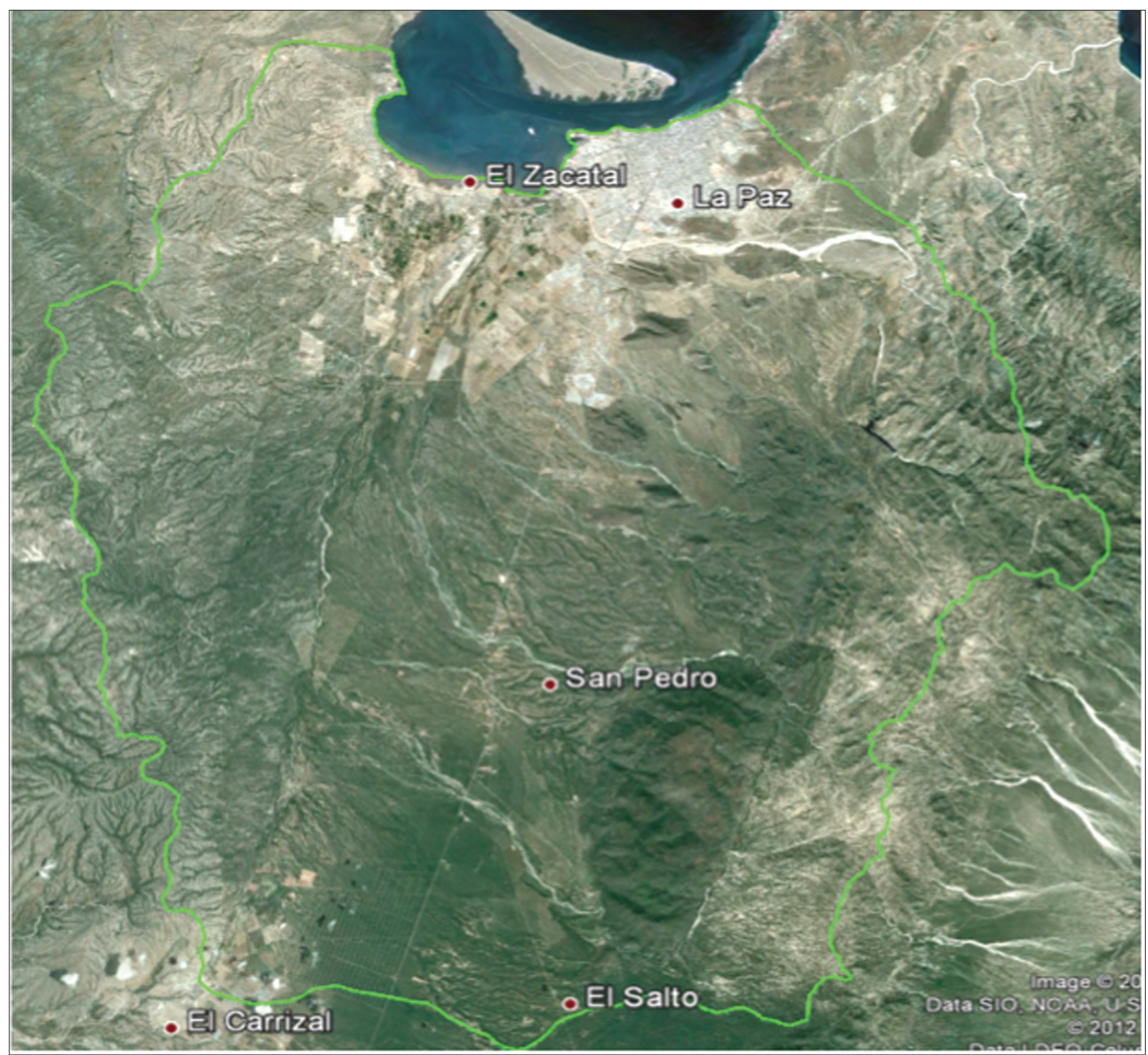

Figura 2. Delimitación de la Cuenca de La Paz y sus poblaciones. 


\section{Material y métodos}

Como propósito global de la ordenación de cuencas está el aumento de la calidad de vida de los habitantes de la zona, a través de todas las mejoras en el medio natural y los recursos naturales de la cuenca; por lo que se deberá intentar en todo momento analizar y estudiar la problemática y el planteamiento de soluciones de una manera "integral", tomando en cuenta que el ámbito analizado es un sistema en el que los diferentes componentes están interrelacionados y que no es conveniente manejar cada componente como si fuera una entidad única.

La Junta de Andalucía, España, en el año de 1993 fue pionera en la formulación de esta idea en documentos político-administrativos para la Ordenación de Cuencas en ámbitos áridos y semiáridos. La subordinación de la gestión de los recursos hidráulicos a las necesidades del desarrollo socioeconómico y a los planteamientos de ordenación territorial, constituye una premisa a aplicar tanto a escala regional como intrarregional. Esto sirvió para plantear los diversos esquemas de ordenación de cuencas, que son basados en la cuantificación y tratamiento de la erosión, por lo que se decidió utilizar el modelo USLE, la metodología con criterios de Mintegui Aguirre y López y por último la metodología con criterios de Sostenibilidad. Cada metodología conservará el uso actual de aquellas superficies en las que las pérdidas de suelo están por debajo del umbral admisible, y por otro lado intentará llevar hasta niveles admisibles aquellos terrenos que son capaces de soportar pérdidas de suelo superiores, ya sea realizando prácticas de conservación en los suelos agrícolas o cambiando el uso del suelo.

\section{Resultados y discusión}

\subsection{Ordenación de la cuenca basada en la metodología USLE}

El modelo de erosión USLE (Ecuación Universal de Pérdida de Suelo) a través de la superposición de distintos mapas (mediante herramientas SIG) relativos a los parámetros que integran la ecuación 1, produce un mapa final de estados erosivos:

$$
A=R \cdot K(L S) \cdot C \cdot P
$$

Donde $A$, es la tasa de pérdidas de suelo $\left(\mathrm{Mg}\right.$. ha $\left.\mathrm{a}^{-1} \cdot \mathrm{año}^{-1}\right)$. La variable $R$ es un factor de la ecuación paramétrica que se relaciona con la erosividad de la lluvia, normalmente plasmado en las líneas iso- $R$ : En el caso de México, dado el escaso número de estaciones con pluviógrafos que existen en el país y con pocos datos previos que agruparan zonas con el mismo factor $R$; se aclara que existe un estudio por parte de Hector Gregorio Torres Cortés para el fondo sectorial CONACYT-CONAGUA que propone información al respecto aunque a una escala que no es conveniente para esta investigación, por lo tanto fue necesario regionalizar las estaciones meteorológicas de la zona de estudio a fin de caracterizar áreas sujetas a condiciones 
similares de lluvia. Se hizo uso de una metodología alternativa para determinar el factor $R$ (FAO-PNUMA-UNESCO, 1980) a partir de la información hidrológica disponible de la Comisión Nacional del Agua, en las 11 estaciones climatológicas distribuidas en el territorio de las cuales se tienen registros mensuales y diarios de precipitación y temperatura desde 1971 hasta la fecha. Ésta técnica se basa en el Índice modificado de Fournier (IMF), realizada por (Arnoldus, 1977) que se muestra en la ecuación 2 de la siguiente manera:

$$
R=\sum_{1}^{12} \frac{\left(P_{1}\right)^{2}}{P}
$$

Donde $R$ es el factor de erosividad de la lluvia, pi, es la precipitación mensual y $P$, es la precipitación anual $(\mathrm{mm})$.

Para el cálculo del factor $K\left(\mathrm{t} \mathrm{m}^{2}\right.$ hora $\left.\mathrm{J}^{-1} \mathrm{~cm}^{-1} \mathrm{ha}^{-1}\right)$, que es relativo a la erosionabilidad e intersección de litofacies. Para el cálculo de los valores del factor K para la zona de estudio se utilizó también la información proporcionada del estudio geohidrológico La Paz-El Carrizal (Subsecretaría de Infraestructura Hidráulica, 1985) además del uso de la herramienta de interpolación Kriging en un modelo SIG.

Mapa de pendientes definidor de LS. Para el cálculo del factor LS en la zona de estudio, fue necesario elaborar el modelo digital de elevaciones (MED) para la cuenca de La Paz. Posteriormente, al contar con los datos anteriores se utiliza el software Usle2D, (Van Oost, K. et al., 2000). Este programa usa la fórmula de (Mc Cool, 1987) para el cálculo del factor LS.

Mapa de Cultivos y Aprovechamientos del suelo: definidor de C y P. Para estimar el Factor $\mathrm{C}$ de cobertura vegetal, se utilizaron las composiciones mensuales del Índice de Vegetación de Diferencia Normalizada (NDVI) mediante el tratamiento de imágenes de satélite proporcionadas por el NOAA-AVHRR (National Oceanic and Atmospheric Administration, NOAA), de acuerdo con las relaciones propuestas por (Garatuza Payán, 2005) generalizadas para las regiones semiáridas del Norte de México, como es el caso del Factor C para Pastizales y Matorral Xerófito que se muestra en la ecuación 3 :

$$
C=0.4304-0.0099 X+0.00006 X^{2}
$$

Donde $X$ es el valor de NDVI para la zona de estudio. Y así subsecuentemente se utilizan ecuaciones propias para los distintos tipos de vegetación (Tab 1) presentes en la cuenca.

Tabla 1. Ecuaciones asignadas para el factor $\mathrm{C}$ en los usos de vegetación para la cuenca de La Paz

\begin{tabular}{|c|c|}
\hline Estrato de vegetación & Factor C \\
\hline Pastizal y matorral xerófito & $0.4304-0.0099 X+0.00006 X^{2}$ \\
\hline Matorral espinoso & $0.2508-0.005 X+0.00003 X^{2}$ \\
\hline Selva baja caducifolia & $0.326-0.0071 X+0.00004 X^{2}$ \\
\hline Pino y encino & $0.3732-0.0084 X+0.00005 X^{2}$ \\
\hline
\end{tabular}


Factor de prácticas de conservación de suelos agrícolas P. En el caso de la cuenca de La Paz no existen algún tipo de prácticas de conservación del suelo, por lo que el factor P, se valoró en 1 para toda la zona de estudio.

Los resultados de la pérdida de suelo en la cuenca de estudio, se reflejan en (Tab. 2) y son definidos en seis categorías, las cuales están acompañadas por el resultado de la distribución superficial de la cuenca en estudio.

Tabla 2. Superficie de pérdidas de suelo o erosión en la Cuenca de La Paz.

\begin{tabular}{|c|c|}
\hline Nivel de erosión (A) & Superficie $\left(\mathrm{km}^{2}\right)$ \\
\hline $\mathrm{A}<10 \mathrm{Mg} \cdot \mathrm{ha}^{-1} \mathrm{año}^{-1}$ & 995.77 \\
\hline $10<\mathrm{A}<25 \mathrm{Mg} \cdot \mathrm{ha}^{-1} \mathrm{año}^{-1}$ & 59.85 \\
\hline $25<\mathrm{A}<50 \mathrm{Mg} \cdot \mathrm{ha}^{-1}$ año $^{-1}$ & 56.54 \\
\hline $50<\mathrm{A}<100 \mathrm{Mg} \cdot \mathrm{ha}^{-1} \mathrm{año}^{-1}$ & 67.15 \\
\hline $100<\mathrm{A}<200 \mathrm{Mg} \cdot \mathrm{ha}^{-1}$ año $^{-1}$ & 29.21 \\
\hline A $>200 \mathrm{Mg} \cdot$ ha $^{-1}$ año $^{-1}$ & 0.94 \\
\hline
\end{tabular}

USLE utiliza baremos de pérdidas de suelo tolerables, At que en este caso son establecidas en $10 \mathrm{Mg}$. ha ${ }^{-1}$ año $^{-1}$ por FAO, PNUMA y UNESCO, y donde A son las pérdidas de suelo genéricas. Por lo que será preciso conservar el uso actual de aquellas superficies en las que las pérdidas de suelo estén por debajo del umbral admisible, y por otro lado intentar llevar hasta niveles admisibles aquellos terrenos que son capaces de soportar pérdidas de suelo superiores, ya sea realizando prácticas de conservación en los suelos agrícolas o cambiando el uso del suelo. Las actuaciones presentadas en (Tab. 3) son las siguientes:

Tabla 3. Superficies de los usos de suelo asignados según el modelo de erosión USLE en la cuenca de La Paz.

\begin{tabular}{|c|c|c|}
\hline Código & Uso futuro & Superficie (km $\mathbf{k m}^{\mathbf{}}$ \\
\hline 1 & Mantener uso agrícola & 63.07 \\
\hline 2 & Mantener uso forestal & 890.42 \\
\hline 3 & Realizar prácticas de conservación de suelos & 0.99 \\
\hline 4 & Repoblación en terreno forestal & 140.24 \\
\hline 5 & Repoblación-Cambio de uso & 90.42 \\
\hline 6 & Improductivo & 36.86 \\
\hline
\end{tabular}

Los resultados de la propuesta de ordenación según el modelo USLE son los que se reflejan en (Fig. 3; Tab. 3). El carácter universal del modelo USLE lo convierte en un buen instrumento para planificar el uso agronómico del territorio. Como contraparte se observa que la ecuación general USLE no incluye el cálculo de la deposición y acumulación de sedimentos (De Roo, 1993). También el modelo USLE 


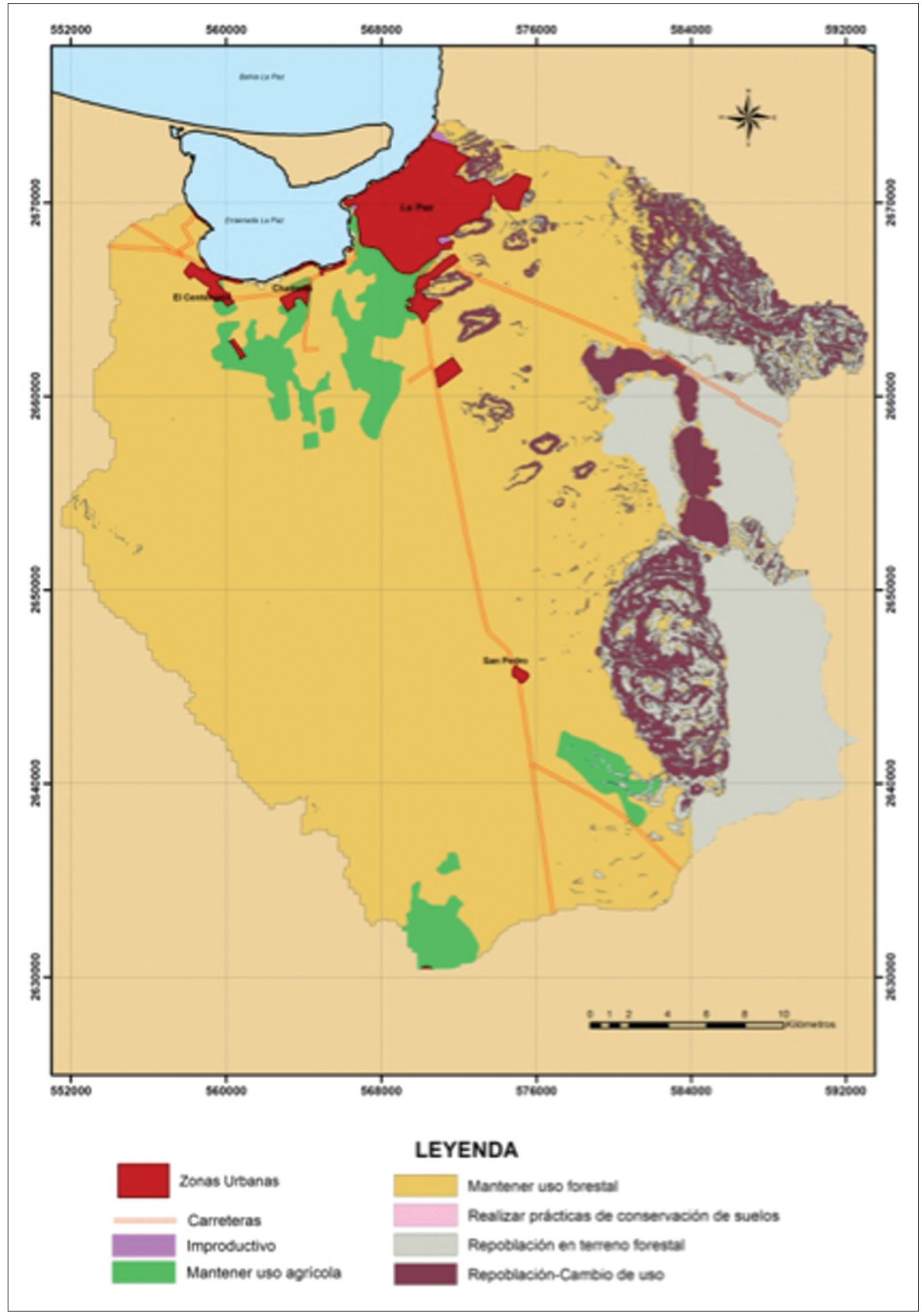

Figura 3. Mapa de Ordenación según USLE. 
hace una clasificación generalista de los usos futuros propuestos, no diferenciando las formaciones vegetales presentes en una cuenca. Será labor del gestor el plantear actividades de uso futuro más específicas a partir de la ordenación.

\subsection{Ordenación de la cuenca basada en la metodología de criterios Mintegui Aguirre y López}

La aplicación de esta metodología se basa primordialmente en los índices de protección del suelo por la vegetación en el modelo USLE y considera la vegetación como el elemento fundamental de gestión en la ordenación de una cuenca hidrográfica. (Mintegui y López 1990).

Se emplea elaborando una superposición de los mapas de pendientes, pérdidas del suelo según el modelo USLE, usos del suelo y mapa de erosionabilidad. A partir de lo anterior para cada polígono con las mismas características, se le asigna un uso futuro del suelo. Por lo que las superficies obtenidas después de realizar la ordenación de la cuenca se recogen en la tabla siguiente.

Tabla 4. Superficies de usos de suelo asignados según el método de ordenación Mintegui Aguirre y López (1990) en la cuenca de La Paz.

\begin{tabular}{|c|c|c|}
\hline Código & Uso futuro & Superficie $\left(\mathbf{k m}^{\mathbf{2}}\right)$ \\
\hline 1 & Conservar cubierta arbórea & 104.43 \\
\hline 2 & Mantener el matorral & 765.98 \\
\hline 3 & Mantener el pastizal & 10.88 \\
\hline 4 & Mantener la cobertura & 19.21 \\
\hline 5 & Conservar cultivos & 57.63 \\
\hline 6 & Mejorar el matorral & 5.97 \\
\hline 7 & Mejorar el pastizal & 0.07 \\
\hline 8 & Repoblación forestal & 204.4 \\
\hline 999 & Improductivo & 53.43 \\
\hline
\end{tabular}

Para el caso de esta metodología cuyos resultados propuestos se encuentran en (Fig. 4; Tab. 4) se destacan fortalezas como el establecimiento de mayor cantidad de alternativas en actividades de ordenación respecto al modelo USLE, tomando en cuenta características del suelo y vocación del territorio. Sin embargo este modelo no tiene en cuenta determinados aspectos de interés para la ordenación de cuencas en este ámbito, como aspectos posicionales o zonificación de la cuenca (áreas dominantes y áreas dominadas), ni la capacidad actual del territorio para permitir un cierto nivel de la serie climática, es decir, no hace mención de las potencialidades bioclimáticas del medio (Tejera, 2001). 


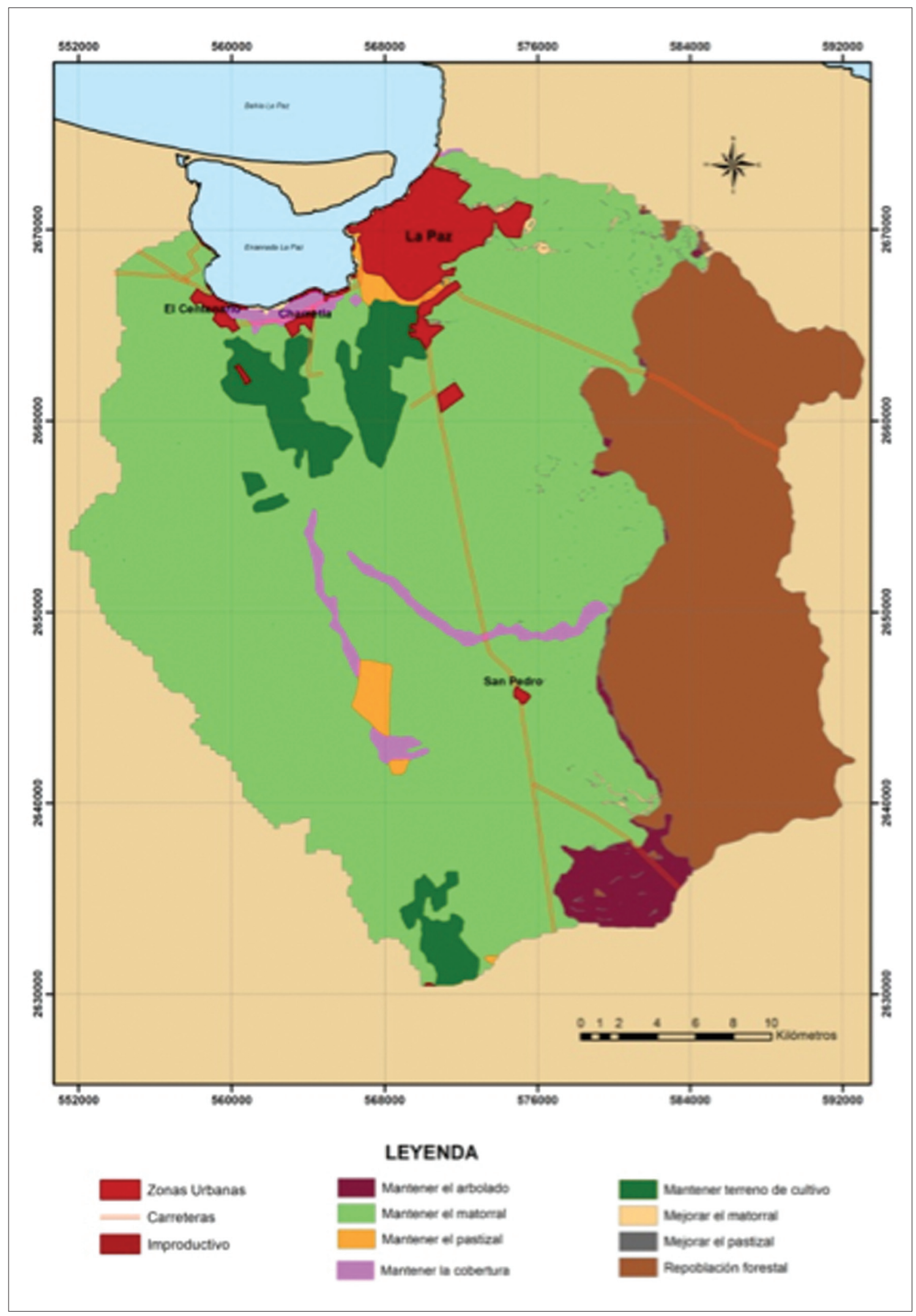

Figura 4. Mapa de Ordenación según criterios de Mintegui Aguirre y López. 


\subsection{Ordenación de la cuenca basada en la metodología de criterios de sostenibilidad (MOCS)}

Esta metodología incorpora los objetivos de la protección hidrológico forestal, los complementarios de la ordenación de cuencas y los de sostenibilidad, apuntando a una visión holística del medio natural alentando el aprovechamiento sostenible de los recursos previo análisis del grado de conveniencia de las actuaciones que se propongan. Los elementos del medio seleccionados para su análisis son, pendientes, influencia hídrica, uso del suelo, litología, pérdidas de suelo, propiedad, protección y espesura de la cubierta arbórea. Las relaciones entre elementos del medio y actividades, desde el punto de vista de asignación de usos del suelo, se concretan en dos conceptos: capacidad y grado de conveniencia. Realizando la superposición con el SIG de las coberturas de adecuación de las actividades propuestas se obtienen las siguientes combinaciones (adecuación alta y media) posibles.

Tabla 5. Superficies de los usos de suelo asignados según metodología de ordenación con criterios de Sostenibilidad en la cuenca de La Paz.

\begin{tabular}{|c|c|c|}
\hline Actividad & Adecuación & Superficie $\mathbf{( k m}^{\mathbf{2}} \mathbf{~}$ \\
\hline Repoblación forestal protectora & Alta & 199.19 \\
& Media & 951.61 \\
\hline Completar espesura en masas & Alta & 106.92 \\
con espesura defectiva & Media & 201.58 \\
\hline Hidrotecnias de corrección & Alta & 0.004 \\
de cauces & Media & 0.062 \\
\hline Restauración de riberas & Alta & 0.52 \\
& Media & 0.088 \\
\hline Repoblaciones para aumentar & Alta & 893.17 \\
la biodiversidad & Media & 89.41 \\
\hline Conservación de enclaves & Alta & 913.29 \\
de interés & Media & 42.69 \\
\hline Acotación de áreas al pastoreo & Alta & 118.009 \\
& Media & 828.02 \\
\hline Mantenimiento y mejora & Alta & 718.07 \\
de matorrales & Media & 12.07 \\
\hline Mantenimiento del uso agrícola & Alta & 57.68 \\
& Media & 0.008 \\
\hline
\end{tabular}

Para la metodología con criterios de sostenibilidad los resultados propuestos se detallan en (Fig. 5; Tab. 5). Existe en este modelo una definición de objetivos clave para la problemática de la cuenca y con ello actividades de ordenación más específicas. Las matrices de capacidad de acogida de las actividades por parte del medio, 


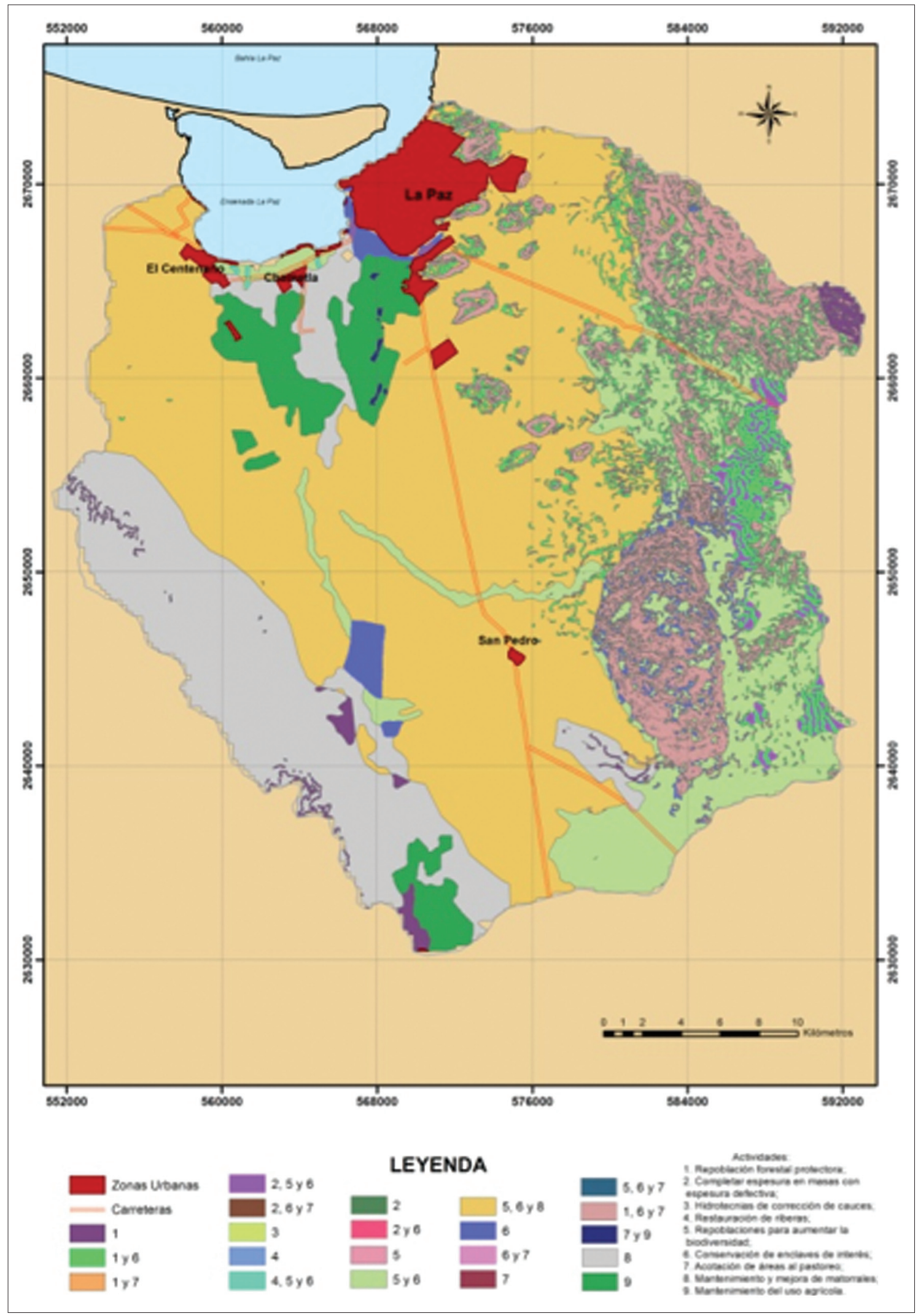

Figura 5. Mapa de Ordenación según criterios de Sostenibilidad. 
son flexibles a incorporación de nuevos índices representativos del medio a analizar. Entre sus carencias, se encuentra la subjetividad en la valoración de las matrices, es decir, estos valores dependerán de la decisión de los gestores. Cabe añadir que su ejecución es más compleja que las otras metodologías.

\section{Conclusiones}

Considerando el análisis comparativo de las metodologías con criterios de Sostenibilidad, Mintegui Aguirre y López, y el modelo USLE, se han podido identificar las ventajas y desventajas de cada una de ellas, lo que lleva a la conclusión de que estas metodologías son complementarias. Las metodologías con criterios de Sostenibilidad y Mintegui Aguirre y López, son técnicas más cualitativas que se basan en los resultados cuantitativos y físicos de pérdidas de suelo del modelo USLE. Se observa que las actividades resultantes presentan alto grado de coincidencia en las superficies de asignación de uso de suelo para la ordenación de la cuenca de La Paz. Presentando una mayor coincidencia entre sí, los modelos USLE y Mintegui Aguirre y López.

Según los resultados de pérdida de suelo, el grado de erosión de la cuenca es de moderado a alto, aunado a esto y con el patrón de lluvias tan azaroso y escaso, principalmente de tipo convectivo y ciclónico, el grado de deforestación de la cuenca es alarmante, lo cual recoge un panorama urgente de atender. El cambio de uso de suelo será una actividad necesaria y urgente para intentar recuperar parte de las zonas degradadas de la cuenca.

Sin embargo se establece que las tres metodologías analizadas no recogen adecuadamente la problemática existente en el ámbito árido de Baja California Sur. Por lo que se considera necesario el desarrollo de nuevos criterios de ordenación y la creación de una metodología de ordenación de cuencas específica para el ámbito biogeográfico de la zona estudiada, que proporcione respuestas a los problemas de degradación y desertificación existentes a nivel de cuenca. Con la elaboración de esta investigación, surgen cuestiones que encuentran respuesta en la creación de una nueva metodología de ordenación de cuencas hidrográficas, que tendrá en cuenta la elaboración de matrices en donde serán añadidos nuevos criterios que engloben tanto la hidrología superficial como la subterránea (influencia directa de la recarga de acuíferos) y los riesgos subsecuentes (inundaciones y aterramiento en embalses) supuestos en la ordenación de un territorio. Esta nueva gestión integrada de cuencas será valiosa al establecer las pautas para la ordenación, gestión del recurso hídrico y recursos naturales en regiones áridas y con alta erosión de la zona desértica del Noroeste de México.

\section{Agradecimientos}

Agradecimiento particular a CONACYT, Consejo Nacional de Ciencia y Tecnología (México). 


\section{Bibliografía}

Arnoldus, H, 1977, Predicting soil losses due to sheet and rill erosion, FAO Conservation Guide 1, Guidelines for watershed management.

Comisión Nacional del Agua [CONAGUA], 2008, Estadísticas del Agua en México. 1ra. Edición 2008, ISBN 978-968-817-895-9.

De Roo, A. P. J. 1993, Modelling surface runoff and soil erosion in catchments using Geographical Information Systems. Validity and applicability of the ANSWERS model in two catchments in the loess area of South Limburg (The Netherlands) and one in Devon (UK), Netherlands Geographical Studies.

FAO-PNUMA-UNESCO, 1980, Metodología provisional para la evaluación de la degradación de los suelos. Organización de las Naciones Unidas para el Desarrollo de la Agricultura y la Alimentación (FAO), Programa de las Naciones Unidas para el Medio Ambiente (PNUMA). Organización de las Naciones para el Medio Ambiente (UNESCO), Roma Italia.

Garatuza P.J., Sánchez R., Sánchez S. y Navarro J., 2005, Using remote sensing to investigate erosion rate variability in a semiarid watershed, due to changes in vegetation cover. Sediments Budgets, I. IAHS Publ. 291.

Instituto Nacional de Estadística y Geografía INEGI, 2010, En México somos 112 millones 322 mil 757 habitantes al 12 de junio de 2010., http://www.inegi.org.mx/inegi/contenidos/espanol/prensa/comunicados/rpcpyv10.asp, Acceso el 15 de Noviembre 2014.

Junta de Andalucía, Consejería de Obras Públicas y Transportes, 1993, Bases para la Política Hidráulica en Andalucía, Sevilla.

Mccool, D. K., Brown, L. C., Foster, G. R., Mutchler, C. K. And Meyer, L. D. 1987, Revised Slope Steepness Factor for the USLE, United States of America.

Mintegui J. A. y López Unzú, 1990, La ordenación agro-hidrológica en la planificación. Servicio de Publicaciones del Gobierno Vasco. Vitoria.

Sánchez Rigoberto, 2004, Características ambientales y áreas potenciales para cultivos en el municipio de La Paz, B.C.S, Instituto Nacional de Investigaciones Forestales, Agrícolas y Pecuarias.

Subsecretaría de infraestructura Hidráulica, 1985, Estudio geohidrológico complementario de las cuencas La Paz-El Carrizal para proporcionar agua en bloque a la ciudad de La Paz B.C.S., Universidad Autónoma de Baja California Sur.

Tejera G. R, 2001, Metodología para la ordenación de cuencas hidrográficas con criterios de sostenibilidad, Tesis Doctoral. Universidad Politécnica de Madrid. Escuela Técnica Superior de Ingenieros de Montes.

Van Oost, K., Govers, G., Desmet, P., 2000, Evaluating the effects of changes in landscape structure on soil erosion by water and tillage, Landscape Ecol. 15(6), 577-589.

Wischmeier, W.H. y D.D. Smith, 1978, Predicting Rainfall Erosion Losses: A Guide to Conservation Planning, Agriculture Handbook No. 537. USDA/Science and Education Administration. US. Govt. Printing Office, Washington, DC.

Z. Flores Emigdio, 1998, Geosudcalifornia, geografía, agua y ciclones, Universidad Autónoma de Baja California Sur, México, D.F. 
